\title{
EDITORIAL
}

\section{Crecimiento demográfico y cambio climático}

El mundo seguirá creciendo. Las más recientes proyecciones de las Naciones Unidas no prevén todavía un punto de inflexión en el crecimiento poblacional en el transcurso del presente siglo, pero se estima que, dadas las tendencias demográficas registradas, para mediados de siglo seremos alrededor de 9 mil 300 millones de habitantes en el planeta. La dinámica demográfica es la principal fuerza conductora del cambio ambiental global, pues constituye una presión para ocupar cada vez más espacios, explotar más recursos, producir más alimentos, consumir más agua, contaminar más, utilizar más energía y emitir más gases de efecto invernadero (1).

La disminución del crecimiento demográfico resulta un requisito necesario para reducir las emisiones de carbono en el futuro; sin embargo, se objeta que ello puede dar lugar a programas de "control demográfico" en los países en desarrollo. Y como se sabe, estos países se verían afectados gravemente por el cambio climático; desde tal perspectiva ha sido identificado por lo menos una de tres posibles formas de interacción entre las tendencias demográficas y los efectos del cambio climático: (i) un rápido deterioro de las fuentes de recursos naturales, (ii) mayor demanda de recursos escasos y (iii) el aumento de la vulnerabilidad humana a fenómenos meteorológicos extremos (2).

Resultados de investigaciones indican que habría que ofrecer más posibilidades de acceso voluntario a los servicios de planificación familiar a las comunidades pobres de los países menos adelantados. Este enfoque prioriza el bienestar de las comunidades pobres afectadas por el cambio climático, a diferencia de la otra postura que propone la reducción del crecimiento de la población a fin de limitar el aumento de las emisiones de carbono mundiales (2).
El crecimiento poblacional aumenta la demanda de bienes y servicios, sin duda implica una mayor presión ambiental. Al mismo tiempo, el crecimiento económico, lo que se considera como la mejor manera de reducir la tasa de crecimiento de la población, también se asocia con una severa degradación de los recursos naturales. Hasta ahora, en los países en desarrollo los mecanismos del crecimiento económico han sido incapaces de reducir la presión demográfica y el desarrollo económico inequitativo, pero a la vez estos mecanismos tienden a reforzar los factores de la degradación ambiental resultante de la actividad económica. Es lo que llamamos la paradoja del crecimiento económico.

Dos soluciones se prevén normalmente para limitar el crecimiento de la población en el tercer mundo: la primera, se basa en el control de la tasa de fecundidad y la otra en el crecimiento económico. En teoría, estas dos soluciones deben ser complementarias, pero en la práctica a menudo son contradictorias. Aunque se han conseguido algunos buenos resultados, la primera solución, basada en el control de la fertilidad, no solo se enfrenta a muchos obstáculos económicos, sociales y culturales que limitan seriamente su aplicación en los países pobres, sino también parece incapaz de hacer frente al tamaño del problema demográfico. La segunda solución, como es el crecimiento económico, cada vez más generalizada, también está abierta a algunas objeciones básicas (3).

Las discusiones sobre el cambio climático generalmente tienden a concentrarse en las emisiones de carbono de una persona durante toda su vida, estas son cuestiones importantes y esenciales considerarlas; sin embargo, un reto adicional al que nos enfrentamos es el crecimiento demográfico y el aumento del consumo global de recursos (4). 
Según el Panel Intergubernamental de Cambio Climático (IPCC, por sus siglas en inglés) se espera que el cambio climático intensifique el estrés actualmente padecido por los recursos hídricos, debido al crecimiento de la población, el cambio económico, los usos de la tierra y, en particular, a la urbanización. A escala regional, los bancos de nieve de montaña, los glaciares y los pequeños casquetes de hielo desempeñan un papel crucial con respecto a la disponibilidad de agua dulce. Según las proyecciones, las pérdidas de masa generalizadas de los glaciares y las reducciones de la cubierta de nieve de los últimos decenios se acelerarían durante el siglo XXI, reduciendo así la disponibilidad de agua y el potencial hidroeléctrico, y alterando la estacionalidad de los flujos en regiones abastecidas de agua de nieve de las principales cordilleras (por ejemplo, Hindu Kush, Himalaya, los Andes), donde vive actualmente más de la sexta parte de la población mundial (5).

El Perú, uno de los países que está siendo afectado por el cambio climático, al 2013 tiene una población de 30475144 habitantes y el departamento de Junín 1331253 (6), un alto porcentaje concentrado en la zona urbana y la población de la zona rural dedicada a la agricultura sería uno de los sectores más afectados por este fenómeno.

Sin duda, el crecimiento poblacional es un factor que acelera el cambio climático, en los Andes Centrales del Perú se puede encontrar varios casos en los cuales este crecimiento impacta negativamente. Es el caso del lago Chinchaycocha, contaminada por relaves mineros y una creciente demanda de explotación de su biodiversidad por las comunidades asentadas en los alrededores. También, está la contaminación del río Mantaro con aguas residuales y residuos sólidos arrojados desde los diversos distritos de la cuenca del Mantaro, sobre todo alta y media, aguas contaminadas que son utilizadas para la generación de energía eléctrica desde la Central Hidroeléctrica Santiago Antúnez de Mayolo, ubicada en el departamento de Huancavelica. Otro caso preocupante es la aceleración del retroceso de la masa glaciar del nevado Huaytapallana, debido al incremento de actividades turísticas, culturales, de pastoreo, entre otras en la zona (7).

\section{Referencias bibliográficas:}

1. Gonzáles DG. Energía y cambio climático. Revista Derecho Ambiental y Ecología. 2013; 10(55): 61-63.

2. Bryant L, Carver L, Butler CD, Anaged A. Climate change and family planning: leastdeveloped countries define the agenda. Bull World Health Organ. 2009; 87: 852-857.

3. Beato F, Chiarello F. Population, environment and economic growth: a sociological perspective. Theomai [Internet]. 2000 junio [citado 21 de mayo de 2013]; 1(1): [1 pantalla]. Disponible en: http://www.redalyc. org/articulo.oa? id = 12400102

4. Center for biological diversity [Internet]. New Mexico: Center for biological diversity; 2012 [citado 14 de julio de 2013]. Página principal; [1 pantalla]. Disponible en: http:// www.biologicaldiversity.org/programs/ population_and_sustainability/climate/

5. IPCC. Climate change 2007: impacts, adaptation and vulnerability. Cambridge: Cambridge University Press; 2007.

6. Instituto Nacional de Estadística e Informática [Internet]. Lima: Instituto Nacional de Estadística e Informática. 2013 agosto [citado 20 de agosto de 2013]; Magnitud y crecimiento poblacional; [1 pantalla]. Disponible en: http://www.inei.gob.pe/ estadisticas/indice-tematico/poblacion-yvivienda/

7. Arroyo J. Impactos de las actividades antrópicas en el nevado Huaytapallana. Apuntes de Ciencia \& Sociedad [Internet]. 2012 mayo [citado 13 de abril de 2013];02(01): [1 1 pantallas]. Disponible en: http://www.revista-apuntes.pe

Wilfredo Bulege

Editor jefe 